\title{
Towards an Inclusive Psychology
}

With this issue, the National Academy of Psychology (NAOP), India in association with Springer India is launching the quarterly journal Psychological Studies in print as well as electronic forms facilitating the journal's accessibility. We felt there was a need to create greater space for effective dialog and exchange of ideas at the international level. In order to streamline the journal's production, an online system for manuscript submission has been introduced. Authors can $\log$ on at $\mathrm{http} / /$ www.editorialmanager.com/psyi for submissions and monitoring the same. This journal was launched in 1956 with the late Professor B. Kuppuswamy as titular and Professor B. Krishnan as Executive Editor at the University of Mysore. Subsequently, it was edited by Professor M.A. Faroqi of the University of Calicut. The journal has steadily progressed over the last six decades. In 2000, the NAOP adopted the journal and since then it has been the official journal of NAOP. The new arrangement of its publication marks the beginning of a new era in this continent as far as professional scientific publication in psychology is concerned.

The mission of this journal is to provide its readers with articles that present and discuss a wide range of issues, findings and practices in psychology for the promotion of an inclusive and culturally relevant scientific psychology. As a peer-reviewed journal, Psychological Studies would serve as an outlet for the dissemination of findings and applications of psychology in its diverse fields. Contributions of various kinds including empirical works, theoretical papers, target articles with peer commentaries, interventions, assessments, case studies, debates and reviews are welcome. We shall also occasionally publish interviews of scholars on important themes. We envision special theme issues and invite suggestions to this end. In terms of methodology, we believe in pluralism and encourage quantitative as well qualitative and interpretive approaches.

It may be noted that modernity has been a major force in the shaping of the discipline. Its emphasis on democracy, liberal individualism, and science enabled the psychological scientists and professionals with a powerful conceptual heuristic. Subsequent developments have contextualized knowledge in a broader framework. Attention is being drawn to the cultural, historical, and linguistic structuring of knowledge and multiplicity of perspectives on knowledge. The critical perspective has brought into centre the relationship between the process of knowledge production and power dynamics in the society. During the last few decades, the culturally informed researches in cross-cultural, cultural and indigenous psychologies have increasingly shown that the EuroAmerican perspective is a special case of culturally rooted indigenous psychology. We, therefore encourage publications which have the potential to enhance our conceptual capital, symbolic resources and methodological know-how by drawing on diverse cultures and disciplines. We believe that such efforts would not only contribute to better theorization but also amplify the applied potential of our studies.

The time has come to explore the possibilities of interdisciplinary approach to psychological processes. Insights from disciplines such as philosophy, biology, neuroscience, computer science, economics, literature, history, sociology, anthropology, cultural and political studies would certainly enhance the scope and depth of teaching and learning. The insularity of our approaches often makes it difficult to learn from our co-travelers from the neighboring academic spaces. This practice often under- and misrepresents the reality. This journal seeks to promote mutual learning and integration through interdisciplinary engagement with the diversity, complexity and continuity of human experiences. We think that an empirical science built on observations taken exclusively from one group is problematic. Yet much of the representation in contemporary psychology is rooted in Western database and the developments of psychology in other parts of the world are often missing. The richness of human experience and cultural variations has remained unnoticed. Therefore, we shall appreciate ideas, theories and conceptual alternatives emanating from indigenous concepts of any culture.

In order to be a psychologist or social scientist of any persuasion one needs to have a thorough understanding of cultural, social and politico-historical processes as well as human subjectivity. An interdisciplinary foundation is must for building capacity to authentically engage with individuals and social systems. The interplay of the 'social' and the 'cultural' with subjectivities situated in the specific politico-historical time and space is highly desirable for having impact of research on social policy. Such a move may require crossing the disparate disciplinary identities 
and trying to create a new abode positioned in such a way that a genuine ground for communication with different disciplines becomes viable. To this end we have to change our mindset for an open and less hierarchical dialog with a critical eye. This would help sharpen the empathic quality and an ethically mature stance in our research orientation.

The journal would endeavor to encourage publications related to underrepresented cultures, communities and groups living on the margins of society. Also, as psychological knowledge has significant consequences for people in different sectors as well as common man we would like to create space for dialog with the society. The authors are urged to use jargon free language which can be easily understood and bridge may be developed between scientific knowledge and society.

This is an exciting time to be associated with the field of psychological studies. The expansion of psychology in its scope, methodology, levels of analysis, linkages with other discipline show many contradictions and confluences. The potential of a broadened perspective is certainly greater than before. Taking the concerns in view we consider that it is important for an inclusive psychology to move toward an exploration and critique that is increasingly more oriented to the larger psychological discipline, to theoretical formulation and to a greater articulation of ambiguities in the interpretation and understanding of the findings. Attaining these goals seems more viable now than it has been in the past. Psychology is becoming a pluralistic enterprise in which a wide range of perspectives are encompassed.

This issue of Psychological Studies contains 7 articles that illustrate the diversity of interest in pursuing psychological research. As the readers will see for themselves, this issue includes contributions from scholars representing different parts of the world. The Target Article by Paranjpe on Indian theory of Rasa with commentaries by peers from philosophy, psychology and anthropology shows the potential of indigenous ideas in the domain of emotion and affect. He presents an analysis of Bhakti Rasa (celestial love) to illustrate how life gets transformed and lifted to higher levels of spirituality. Marsella, a long time champion of inclusive psychology, critically examines the state of affairs in the discipline of psychology. He notes that the indiscriminate acceptance and application of Western psychological knowledge and practices is a serious abuse for non-Western people and for ethnic/racial minorities in Western nations. We need to acknowledge, develop, and transmit the diverse indigenous/national psychologies from across the world. Bernardo, Ouano, and Salanga present
Filipino learners' concept of academic emotions. The interpretive analysis of the words used to describe emotional experiences associated with learning suggests that Filipino learners' concepts of academic emotions include appraisals of cognitive and even physical conditions, beyond the typical affective conditions assumed in theories of emotions and academic emotions. The article by Das presents conceptualization of intelligence in a fresh perspective. $\mathrm{He}$ observes that cognitive processing is a common base for cognitive theories of intelligence in both the East (India) and in the West (Europe and America). He notes that intelligence is about awareness and the means of achieving a pure state of awareness through self-directed attention to internal thoughts, rather than external objects. Das proposes that using introspective reflection as a tool to explore consciousness is pertinent. Gaschler and Frensch report an experimental test of the Information-Reduction Hypothesis and provide evidence that practice not only affects how information is processed, but also which information is processed. The results imply that Information Reduction is at least in part a consequence of top-down, voluntary control. Dixit and Mohanty focus on understanding of the cognitive processes involved in appreciation of history in a developmental context. They note that appreciation of the difference between past and history, chronology, and historical imagination emerged earlier than the dimensions such as empathy and critical analysis. Finally, Singh and Srivastava examine the relationship between certain individual level determinants of interpersonal trust and its impact on organizational citizenship behavior. They also show that interpersonal trust partially mediates the relationship between individual level factors and organizational citizenship behavior. Interpersonal trust may be used as a strategy to motivate employees to engage in extra role behaviors to ensure improved individual and organizational performance.

We seek the cooperation of the scholarly community to join us in making Psychological Studies a leading journal in the field of psychology. As an Editor I look forward to working with associate editors, the panel of consulting editors, reviewers, and the many authors who will submit manuscripts to the journal. It is their support which will facilitate the development of Psychological Studies and the enhancement of its potential to contribute to human knowledge and making this world a better place. We are optimistic that Psychological Studies will become a stimulating and innovative forum for improvement of the knowledge and practice of psychology.

Girishwar Misra Editor 